\title{
Generating Implications for Design through Design Research
}

\author{
Corina Sas \\ Lancaster University \\ Lancaster, UK \\ corina@comp.lancs.ac.uk
}

\author{
Steve Whittaker \\ University of California \\ at Santa Cruz \\ Santa Cruz, CA, USA \\ swhittak@ucsc.edu
}

\author{
Steven Dow, Jodi Forlizzi, \\ John Zimmerman \\ Carnegie Mellon University \\ Pittsburgh, PA, USA \\ spdow, forlizzi, johnz@cs.emu.edu
}

\begin{abstract}
A central tenet of HCI is that technology should be usercentric, with designs being based around social science findings about users. Nevertheless a repeated but critical challenge in design is translating empirical findings into actionable ideas that inform design, or generating implications for design. Despite various design methods aiming to bridge this gap, knowledge informing design is still seen as problematic. However there has been little empirical exploration into what design researchers understand by such design knowledge, the functions and principles behind their creation. We report on interviews with twelve expert HCI design researchers probing the roles and types of design implications, and the process of generating and evaluating them. We synthesize different types of design implications into a framework to guide their generation. Our findings identify a broader range than previously described, additional sources and heuristics supporting their development as well some important evaluation criteria. We discuss the value of these findings for interaction design research.
\end{abstract}

\section{Author Keywords}

Design knowledge; Implications for design; Design research.

\section{ACM Classification Keywords}

H.5.m. Information interfaces and presentation (e.g., HCI): Miscellaneous.

\section{General Terms}

Design; Theory.

\section{INTRODUCTION}

A central tenet of HCI is that technology should be usercentric, with designs being based around social science findings about users. Nevertheless an outstanding challenge in interaction design is translating empirical findings into ideas or knowledge that inform design, aka generating implications for design $[10,11,23]$. This has led to a perceived gap between empirical studies and design $[1,10,11,20,23,26,28]$. In the HCI field, design researchers often attempt to bridge that gap by describing how empirical findings might influence system explorations. For those who follow this practice, implications are crucial, yet diffi- cult to create and not well understood. This is reflected in a surprising lack of empirical studies investigating implications, although there are critiques of their perceived abstract quality [33]. This gap between fieldwork and design is a paradox given the wealth of practical design methods developed to mine empirical findings to generate actionable design suggestions. Examples include contextual design [4], activity-based prototyping [17] or design sketches [28].

This paper presents interviews with design researchers who employ user-centric design methods. These researchers have evolved practices to bridge the gap because their research products involve building research prototypes that are motivated by fieldwork. We explore these researchers' understanding of implications for design and the principles behind the practice of generating them. One goal is to help other design researchers bridge the gap through a better understanding of different types of implications commonly employed, the sources for generating them and criteria used for evaluating them. We also provide theoretical and practical implications of such knowledge for HCI researchers designing and building prototypes. While previous work has focused on the theoretical debate about the relationship between ethnography and design [13], our work takes a more pragmatic approach to bridging the gap from qualitative fieldwork studies to design. Increased understanding of the largely tacit and challenging process of generating implications should enable design researchers to refine design methods and improve resulting technologies. We analyze fieldwork data to address the following questions:

- What types of design implications are generated through fieldwork? Are they predominantly communicating devices or are there new types such as abstract functionalities of classes of systems?

- What sources of information are used in generating design implications? Are implications predominantly informed by fieldwork data, human science theories and design practice, or are there other sources yet to be discovered?

- How are implications for design generated? What heuristics do people employ? Are implications generated after the analysis of fieldwork data, or during it?

- What are the main characteristics of design implications and how are they evaluated? Do they meet the validity criteria of scientific knowledge, or more specific criteria related to design practice? 
We report an empirical study involving interviews with twelve experienced design researchers. Our contribution consists of a vocabulary to discuss various types of design implications, their sources and evaluation criteria.

\section{RELATED WORK}

\section{Types and Sources of Design Implications}

Implications for design are a specific type of design knowledge for which prior work has referenced three contrasting sources such as fieldwork-, design-, and human science-informed design knowledge. These three different approaches and their specific types of design knowledge are reviewed below including a mixture of fundamental HCItextbook knowledge, with more detailed accounts of the lesser known ones. While we acknowledge the distinct value of each of these three approaches, this paper focuses mostly on the design implications derived from qualitative fieldwork as it is more commonly employed in user-centered design. Additional hermeneutical [34] and aesthetic [17] approaches to design are beyond the scope of this paper.

\section{Fieldwork-Informed Design Knowledge}

\section{Requirements}

Requirements gathering is a common HCI method for generating design knowledge, but it has been critiqued for its failure to capture the richness of social settings $[10,11,23]$. Requirements are highly specific design knowledge derived from fieldwork to support a situated design for that setting. They are intended to capture system goals and functionalities that lead to incremental improvements to existing systems in well-understood contexts. Requirements are highly prescriptive and implementable but are difficult to generalize beyond the settings where they have been explored.

\section{Thick Descriptions}

Ethnographic methods emerged to address the limitations of requirements gathering methods and are typically used in settings where complex social factors are at play. The explicit aim of ethnographic methods is the understanding of socialities, and more elusively to explore how technology may address their key social issues [22]. They typically use analytic methods that generate rich descriptions of social settings, while the process of deriving technical implications from these descriptions is complex and controversial [22]. Controversies concern whether ethnographies should be strictly descriptive or should speak to technology implications [13]. There is limited consensus about how ethnographic work should be expressed as design knowledge, or how that knowledge might be evaluated.

\section{Communicating Devices}

Design knowledge also serves to communicating succinctly to designers the essential fieldwork findings, i.e. personas and scenarios. Scenarios capture narratives describing users' activities in terms of system goals, and users' context of activity [8]. They are described in user-language and tend to be highly situated. Scenarios are versatile concepts which characterize various points on the continuum from fieldwork to design. Carroll [8] identified several functions they could serve, from mere descriptions supporting requirements analysis, to envisionment of conceptual models. Cooper's [9] personas represent archetypal users who manifest critical concrete behaviors, abstracted from multiple users along with engaging personal details to bring the character to life. Their value is partly derived from people's ability to engage meaningfully with fictional characters [19]. Beside provoking and inspiring, personas emphasize situated rather than generalized users, increasing designers' understanding and empathy.

\section{Sensitizing Concepts}

Sensitizing concepts highlight relevant social aspects concerning technology use. The term is borrowed from the social sciences [5], to describe new, intriguing, and underdeveloped observations about significant social problems that new technologies should address $[10,23]$. They serve as interpretative devices for the exploration of empirical instances and for abstracting their common features. More specifically, such concepts provide rich descriptions of socialities that capture key situational features and social interactions [13,33], which potentially orient designers towards key problems [23]. Sensitizing concepts also challenge assumptions about design [23,33], and provide new design research agendas [10]. The abstract nature of sensitizing concepts promotes generalizability but this is counterbalanced by a lack of clarity and limited specification of technologically implementable knowledge [10]. Despite their valuable contributions, ethnographers have repeatedly documented how sensitizing concepts are notoriously difficult to translate into specific or prescriptive design implications $[10,23,26,28,33]$. Finally, proponents of sensitizing concepts do not discuss explicitly their evaluation. In the design context, an implicit criterion is generativity, i.e. the extent to which they lead to novel designs.

\section{Conceptual Models}

Conceptual models introduced by Norman [27], capture users' understanding of a computer system. They are generated at the ideation stage of design to translate requirements into abstract user-centric descriptions of the system [3]. They tend to be generalizable, as alternative conceptual designs allow for a wider exploration of the design space. Conceptual models can also be generated through reflection on developed systems to extract the core features of their success, as further described.

\section{Practice-Informed Design Knowledge Design Concepts}

A different approach to generating design concepts is to critically analyze successful systems to try to replicate their successes using related design concepts [7]. Early examples of successful designs included WYSIWYG desktop metaphor and document editors where important digital objects were rendered on the screen, behaving approximately like real world objects allowing direct manipulation of those objects [24]. More recent design concept approaches include Höök's strong concepts [21], which are general properties that multiple systems might possess. In Höök's work 
these properties include social navigation: socially enabling applications to show for example how users have traversed an information space. A different approach has been taken by Gaver [17], who suggests that designs be motivated by their ambiguity, as they are deliberately underspecified. Because of their focus, the relation between design concepts and prior empirical fieldwork is unspecified. Although the goal of design concepts is clearly to devise systems that are intended to motivate rich interaction experiences, neither Gaver nor Höök provide motivating fieldwork that maps concepts or principles to originating user behaviors. In the same way, no explicit criteria are developed for evaluating design concepts, although the implicit criterion seems to be whether concepts are generative: leading to multiple new instances of the design concept.

\section{Design Heuristics}

Heuristics are simple design-oriented rules of thumb to guide practical system design. Although their exact provenance is unspecified they seem to be derived from designers' expertise and reflections on successful prior practice of system building [12]. Unlike design concepts, their goal is to incrementally improve the design of specific systems, rather than to generate new systems. While less abstract than design concepts and usually depicted in terms of technology properties, heuristics can still vary in their level of abstraction but maintain their generalizability for a class of technologies. They serve as general orienting principles for system design: e.g. Nielsen's first principle of visibility of system status, or Schneiderman's reversibility principle. There have been various critiques of usability heuristics and their application, from being unreplicable or conflating important with more trivial types of system faults [18]. Nevertheless these techniques are commonly used in practical contexts when developing interfaces or interactive experiences and there is a specific system to be evaluated.

Other approaches to design, informed by practice consist of critical design [16], research-through-design [15,36], or material-driven design [34]. Additional types of knowledge derived from practice include design deliberations around issues arising during the design process consisting of responses proposed and their rationale [29], or patterns capturing proven solution to a specific design problem which together allow for the development of a pattern language [2].

\section{Human Science-Informed Design Principles}

A rather different approach to generating design knowledge is to derive design principles from prior social science theory. Carroll [6] identified claims about users' needs that could be informed by social and behavioral theories. Design principles provide high level technology goals [3,12] and tend to be abstract and general, i.e. widely applicable to a broader class of systems. Design principles also tend to be described in people- rather than technology- language, and need to be interpreted by designers to either develop new system types or incrementally improve existing systems. Principles are often evaluated by ascertaining the utility of systems designed after applying the principles. However the mapping between principles and their application to system design can be indirect, making their exact utility difficult to determine. A classic example of human science inspired design principles is the application of Fitts' law concerning perception and action, which has been successfully used in the design of many different types of pointing and input devices. Social sciences have also been used in the design of community systems, exploiting concepts of social capital, self-presentation, and social loafing.

\section{METHOD}

We examined the different types of design implications by employing a common HCI method of interviewing experts, in this case design researchers. For example, Zimmerman and colleagues [36] also interviewed researchers to explore the sources and outcomes of design research. We interviewed twelve experienced design researchers working in HCI, 9 with at least 10 years of experience, and 6 over 20 years, including 2 ACM SigCHI Academy members. Two non-random sampling techniques were employed. Through purposive sampling, invitations were sent to 25 design researchers, with recognized positions in the field, i.e. h-index above 10 , and their expertise in qualitative research methods. A subsequent snowball sampling emerged, with 4 researchers being referred by the initially invited ones. The sample consists of 8 researchers working in academia and 4 in industry research labs with 8 having experience of working in both sectors (Table 1). Eight participants work in the UK, 3 in the US, 1 in The Netherlands, and the sample was gender balanced, with research interests in memory technologies, interaction design, UX and CSCW.

\begin{tabular}{|l|l|c|l|}
\hline$\#$ & \multicolumn{1}{|c|}{ PhD area } & Years expertise & \multicolumn{1}{|c|}{ Current position } \\
\hline P1 & Cognitive Sciences & 25 & Principal Researcher \\
\hline P2 & Cognitive Sciences & 22 & Professor \\
\hline P3 & Computer Science & 24 & Senior Researcher \\
\hline P4 & Interaction Design & 22 & Associate Professor \\
\hline P5 & Information Systems & 20 & Associate Professor \\
\hline P6 & Cognitive Psychology & 20 & Senior Researcher \\
\hline P7 & Informatics & 15 & Professor \\
\hline P8 & Design & 14 & Associate Professor \\
\hline P9 & Education & 10 & Professor \\
\hline P10 & Computer Science & 9 & Associate Professor \\
\hline P11 & Communication Science & 7 & Associate Professor \\
\hline P12 & Psychology & 5 & Researcher \\
\hline
\end{tabular}

Table 1. Participants' profiles

All participants have experiences in designing and building HCI prototypes, so that their own definitions of design implications are derived through years of practice. All participants have strong .expertise in qualitative research methods including ethnography, interviews, diary studies, contextual inquiry as well as evaluation of deployed systems. The interviews lasted from 45 to $120 \mathrm{~min}$ (average $65 \mathrm{~min}$ ), and were semi-structured around a set of core questions. We asked participants: What are design implications? and What 
are their most important roles? We also asked: Are there different types of design implications? and how are they generated: Could you describe the process of generating them from fieldwork studies? Are there any specific information sources you use? Are there fieldwork findings that are more useful to generating implications than others and why? We also explored the main characteristics and criteria used for evaluating design implications: What kind of criteria you use to evaluate implications?

The interviews were completely transcribed, and over 1300 quotes capturing implications were thematically coded and analyzed. The analysis was intensely iterative and collaborative. A conceptual framework developed from prior literature provided initial categories of types of design knowledge and their information sources. This was further refined as new codes emerged to capture specific abstract types of implications, and additional sources, heuristics and evaluation criteria. The authors met at least weekly for over two months to discuss and revise the coding scheme and ensure its validity and reliability.

\section{RESULTS}

\section{Types of Implications for Design}

An important finding is a broader set of design knowledge capturing design implications beyond descriptions, requirements, principles and sensitizing concepts, to include more innovative ones such as meta-abstractions and instantiations. In contrast with ethnographic research emphasizing "thick descriptions" and HCI textbooks highlighting communicating devices, i.e. personas and scenarios, our findings show limited evidence of these types but a strong interest in "short descriptions" of fieldwork data. Our findings reveal a taxonomy of implications for design consisting of short descriptions (27\%), prescriptions (18\%), abstractions and meta-abstractions (23\%), sensitizing concepts $(18 \%)$, and instantiations $(14 \%)$ and their weights in the overall data (totaling 100\%). While descriptions are linked to the design problem explored through the fieldwork, and prescriptions are linked to the design solution, the last four types capture design concepts developed to specifically bridge the gap between the fieldwork and design.

Design Concepts as Abstractions of Technology Functionalities Abstractions of technology functionalities capture general functionalities of an existing class of technologies (P1,P2,P4,P5,P6,P8,P10,P12). They are shaped as suggestions for interpreting more abstract technology goals captured by sensitizing concepts. For example, abstractions capture more technologically-oriented information such as interaction modes or system components: "looking at how people read from paper, you can come up with a set of guidelines like "to support the reading of multiple documents when you are building systems for electronic reading". Another general one would be "to support note taking or the ability to annotate as people read" (P1).

A particular type of abstraction is meta-abstractions focusing on the key aspects of a new class of technologies. They differ from abstract functionalities by moving beyond existing technologies, towards discovering new classes. Metaabstractions offer " a conceptual framework of reference for a new class of technologies [because] it's not about the design of the specific technology but about the whole space like a narrower field within HCI" (P1). Usually, products of a large body of work, meta-abstractions are particularly visionary, enabling shifts in HCI research areas. They aim of transforming thinking about current technologies (P1,P6,P12), by challenging design assumptions and helping designers gain new perspectives: "it's about challenging people's assumptions and [being] critical of some of the more conventional approaches to building these kinds of systems" (P1). For this, they need to be inspiring and motivating: "they not just teach how to think about a class of technology but inspire building new concepts" (P1).

\section{Design Concepts as Instantiations of Abstractions}

Instantiations take the form of possible design concepts embodying an abstract property or feature (P2,P5,P6,P9). Their main aim is to support the understanding of the related abstraction: "We've collected all this data [...] and here are three ideas about systems that might allow people to socially network in several ways; and you would actually describe prototypes that could be implemented" (P2). Instantiations or design exemplars should also stimulate designers to think about the abstract principles they embody to further explore the design space: "you could take those principles and generate a whole bunch of different types of systems that adhere to those principles but are innovative and make other people think" (P2). Such exemplars are described predominantly verbally, and are not actual design solutions, but may act as placeholders within the design space. However they can be realised in working exemplar prototypes. Working exemplars are inspirational as they can be directly understood and used by technologists: "We built systems based on early papers, and system builders have come up to me and said: "I love the xxx system, it was a big inspiration" or "I thought this was really interesting. I wanted to do something like this" (P2). These systems could then serve as design concepts for others who are interested in building similar systems.

Both abstractions and instantiations express underlying design principles derived from fieldwork data, but manifest these differently: as guiding principles or as concrete examples. Abstractions of functionality use fieldwork to derive general principles that systems should possess, without specifying actual instances of systems. In contrast, instantiations specify concrete exemplar systems that could be or have been built. They are intended to be generative of designs that actually get built.

Sensitizing Concepts as Socially-Oriented Design Concepts Sensitizing concepts offer a preferred form of generalized design knowledge for moving beyond the situatedness of requirements (P1,P7,P8,P10,P11). Described in useroriented language, they capture abstract design knowledge. 
They tend to state technology goals generated to meet specific social needs. For this, sensitizing concepts focus on the key aspects of social behaviors identifying key features of the design space, such as: "high level things in terms of a social arrangements impacting design, configurations of families for example and their interrelationships and power structures; and try to articulate them as features of a design space to orient towards" (P10). These social aspects relate to technologies by suggesting a range of social values, such as "different types of moral and ethical sensibility, value constructs to take into account" (P10). Sensitizing concepts often capture intriguing, underdeveloped social concepts which lack clear definition. Thus, they are more open and inspirational than abstract functionalities but less technologically actionable. Therefore, they require additional work from designer to interpret and apply them to specific settings: "I often worry that some implications are too general to give any good guidance from a practical perspective" (P1). Sensitizing concepts are also intended to challenge designers to reflect on how to implement them, and to support the advance of design thinking as they: "might inspire designers or make them think more broadly about a better technology" (P1). Abstractions can be employed to explore aspects of the sensitizing concepts that could become technologically actionable. The following example suggests that the sensitizing concept of privacy can be operationalized into abstract functionalities of a specific system: "if you're building a system concerned with health, then privacy of data is a key consideration so you might state some abstract functions relating to privacy that that class of system should have" (P2).

\section{Succinct Descriptions for Communicating Core Findings}

The most frequent type of design implications derived from fieldwork data consists of short descriptions as summaries of key empirical findings capturing constraints characterizing the problem space that can be used to inform the design space. Often the initial step in the development of the other types of design knowledge, short descriptions are derived through synthesis and abstraction of rich interview- or ethnography-based data: "[to summarize thick descriptions] you need to be able to communicate the most salient parts of it" (P9). They can be user values, context, tasks or concerns expressed through a couple of paragraphs or visual aids. Short descriptions have several roles such as communicating the most relevant findings to allow designers' understanding of the problem context, and inspire them to explore new solutions. More specifically, they serve as communication devices, offering: "a language for for communicating ideas" (P12). Communication focuses on the key fieldwork findings for understanding the design setting, as "their main goal is the understanding of a particular phenomenon" (P11). Such short descriptions also provide explanations for the saliency of the findings and their potential relevance to design: "it's useful to illustrate why a certain finding is important and implications of design is sort of one way of concretizing those" (P6). By promoting such understanding, short descriptions may inspire design- ers to frame the problem or focus on unexplored issues: "making clear what are still open issues which may give some inspiration for designs but are not yet detailed enough to start the design" (P11). The above roles are enabled by the short descriptions' properties of being succinct and highly situated. These strengths are counterbalanced by their limited amount of knowledge that can be technologically implemented or generalizable across settings.

Prescriptions as Requirements for Specific Implementations Another common type of design knowledge is concrete suggestions for simple implementations. These are very specific, prescriptive and easy to implement (P1,P6,P7,P10,P12). Similar to user requirements, prescriptions capture key system properties suggesting how they could be implemented in a particular design: "very specific, design features that would be very easy to address" (P6). Not surprising, they are highly situated and actionable: "something very specific that people can take on board and implement right away" (P1), and have limited power to generalize to settings different that the one investigated.

\section{Examples of Design Implications}

Below are examples for the different types of design implications related to the concept of social awareness (Table 2).

\begin{tabular}{cl}
\hline $\begin{array}{c}\text { Design im- } \\
\text { plication }\end{array}$ & \multicolumn{1}{c}{ Example } \\
\hline & $\begin{array}{l}\text { An influential example is social awareness [14], } \\
\text { capturing understanding of the activities of others } \\
\text { in the context of groupwork, i.e. seamless medi- }\end{array}$ \\
$\begin{array}{l}\text { Sensitizing } \\
\text { concept interaction and sharing of documents. This } \\
\text { spawned a huge amount of subsequent research } \\
\text { that sought to further empirically characterize } \\
\text { social awareness and to build technologies that } \\
\text { would support it [25]. }\end{array}$ \\
\hline
\end{tabular}
An abstract functionality of social awareness is Abstraction extending awareness of remote objects to ges\& tures at, and around them [25]. An example of Meta- meta-abstraction related to social awareness is extending the collaborative tasks from desktop and shared displays to a different class of technologies, i.e. shared tangible interfaces [35].

An instantiation of the abstract property of seamless mediated interaction and sharing of

Instantiation documents was the Agora system itself [25], as one possible design solution. conjunction with desk cameras for capturing users' gestures at remote locations [25].

Table 2. Examples of design implications for social awareness

\section{Sources for Generating Implications for Design}

An important finding is that in generating design implications, design researchers draw from additional sources of information than previously mentioned. These new sources include emerging technology, important social categories and corpus of previous design implications. 


\section{Emerging Technology Context}

A new, important source for generating design implications consists of the technology context (P1,P2,P3,P6,P7,P10,P11). Participants repeatedly pointed out that such context is critical for framing the fieldwork and observed social phenomena (P2, P3): "my sense of the technology is already determining what kind of stuff I would be looking at and human situations I am interested in" (P3). This also includes an awareness of the technological space in terms of shortcomings and possibilities: "being aware of the technology landscape is very important" (P1), with technology affordances being implicitly used as a lens for analyzing the data: "there is an understanding of the basic technological capabilities beforehand" (P6). In an attempt to overcome lack of technical expertise, social scientists may engage in generating design implications with deliberate disregard for technological constraints: "without having the technology in mind but rather thinking about what experience should be facilitated" (P11). This is a critical finding suggesting that even when lacking awareness of the technological context, technology is a significant lens for observing and interpreting data. Such technology context may play different roles from framing ideas to narrowing the scope of the fieldwork (P3,P7,P10): "[to] a specific techno arena that you are exploring through fieldwork, otherwise that is very ungrounded and you have a difficult time in making implications" (P10). Technology as a source for generating implications is an important finding suggesting a critical factor in evaluating what are important observations in the emerging technology context. This is different from other technology oriented approaches such as those based on design concepts $[17,21]$. Our participants were not focusing on successful prior designs but on the general context of emerging technologies. These observations are interesting because the process of generating design knowledge can be seen here as being run backwards, so that technology context is used to frame interpretations of the fieldwork data which are then expressed as technology-oriented design implications.

\section{Social Categories: Social Values, Space and Time}

Another source of information for generating design implications includes aspects of the socialities, such as social values: "focused on different audiences, types of ethical sensibility or value constructs" (P10). These may be data driven, or solely informed by general aspects of social science. They could also include the broader context with reference to social locality and time: "if you are in a park [is important that the technology] is portable so that is a finding [motivating you] to imagine possibilities" (P4), like "what would you say about time in relation of your project that other designers should know about?"(P8).

Previous Fieldwork-Informed Corpora of Design Implications Sets of previous fieldwork-informed design implications offer another source for generating new design implications. In this way, researchers could scrutinize, build on and further extend them: "implications need to be framed in such a way that I can use them as a basis for my own re- search, that I can build on them and that might be either to ratify or actually critique them" (P10).

\section{Fieldwork Data}

Less surprising is that relevant fieldwork findings are the most common source of design implications. This aligns with the standard view on fieldwork-informed design knowledge. What is interesting, is the specific findings acting as sources, such as people's behaviors and preferences (P2), challenges that people face (P5); consistent themes throughout the data (P2); emotionally charged themes $(\mathrm{P} 3, \mathrm{P} 4)$; unexpected findings $(\mathrm{P} 4, \mathrm{P} 7)$ or expected things that did not show up (P8).

\section{Design Practice}

Design implications can also capture lessons learned after the evaluation of technologically implemented design solutions (P1, P10, P11): "We developed a system and then we evaluated it and within that work the implications for design really got better." (P10). This source for generating design knowledge is arguably the most difficult to tap into, because of the challenges of building the designed system in the first place, and the difficulties of evaluating them in real settings: "making the argument that this tool will work in real situations is extremely difficult to show" (P7). Such evaluation builds on actionable knowledge embedded in the designed technologies. Apart from design concepts, another specific type of design knowledge tapping into this source is meta-abstractions. They tend to be the result of a sustained research effort within a domain, consisting of a substantial body of work. This could include iterative fieldwork, development and evaluation of prototypes which facilitates a continual revision of the design knowledge and increases confidence in their validity and generalizability: "We have done a lot of work in our group about different types of xxx technologies, and therefore if you think about these as a class then you can start to sketch out a conceptual framework which will take people thinking about it much more broadly and diversely" (P1).

\section{Human Science Theories}

Consistent with other theory-based approaches to HCI, some participants focused on human science theory when developing design knowledge. These kernel theories are usually outside the HCI area and include social science theories of human behavior or "biological underpinning" (P2): "you have a sort of an inkling that is interesting and you need to go to some kind of social or human theory to figure out what is the literacy of these specific examples" (P3).

How Do Researchers Generate Implications for Design? In generating design implications, researchers are guided by several heuristics. These are employed during analysis of fieldwork data, after analysis, or even before designing the fieldwork study.

\section{Heuristics for Generating Design Implications}

Our participants employ several heuristics when generating design knowledge, including identifying and using prior examples of good implications (P8), as well as distancing 
from the data to take new perspectives. Awareness of cutting edge technologies helps to frame thinking while opening up new possibilities for design: "the designer in our group regularly sends a newsletter where he kind of scours the web looking for new ideas, technologies, wacky things people are working on [...] having that as another set of inputs into the process is really helpful because it gets you out of the rut of thinking of the same old technologies and ideas" (P1). It is an important point that design implications involve more than collecting rich user data. They involve characterizing the technology space, then combining that with the user data. This is critical in order to move beyond 'the same old technologies'. Participants also identified various mapping tactics that help bridge the gap from fieldwork data to design practice. A common form of mapping is from observed behavior to sensitizing concepts (P2,P11), from observed behavior to abstract functionalities and instantiations (P1,P2), and from technology affordances to sensitizing concepts (P6).

\section{After Analysis of Fieldwork Data}

Throughout the process of generating design knowledge, design researchers employ two contrasting approaches to the order of fieldwork data analysis and generation of implications for design. One approach favors the traditional process of generating design knowledge following analysis of the fieldwork data. Participants gave numerous accounts of this classical 'fieldwork first' approach emphasizing the importance of first completing the data analysis before thinking about design implications (P5,P10,P11,P12): "it follows on from having done full analytical work" (P10).

\section{During Analysis of Fieldwork Data}

A rather different approach argues for exploring design implications during data analysis (P1,P4,P6): "it goes hand in hand because while I analyze my data and I see these themes emerging, that automatically triggers an idea of what is the implication for design" (P4). In this respect, this may influence the data reporting and analysis process, as considering design implications during analysis offers an additional lens for identifying the salient findings: "[my analysis] is done with an eye towards design and technology [...] something is interesting because it has some technological implication in which case I might draw that out" (P1). This second approach however, does not abandon the first 'fieldwork first' approach, because participants acknowledged that design implications can be further improved once the findings are properly summarized: "[The fieldwork findings] might be informed by an understanding of the kinds of implications that you want [...] but it becomes easy to think about implications after articulating a clear set of findings" (P10). This quote stresses the iterative nature of the process of generating implications for design.

\section{Before Designing the Field Study}

A few participants employed an even more radical approach by considering design implications before planning the fieldwork: "implications are actually the starting point of research, as in many cases the researcher has an idea"
(P7). For example, people may start from specific technology ideas for which they would like to explore relevant social settings: "The study was motivated by the idea of a graphical user interface that would allow to look at your digital mementos [...] In the end, a design implication was that the screen was not the right thing to do but I was aware what I wanted to find in the end." (P4). However, despite having prior technological framings, participants stressed the importance of staying open to counterintuitive fieldwork data. "I have technology in mind when I select the people and the situations but when I go out, I'm usually just trying to get people to talk and trying to see what they do" (P4). Various research methods contribute differently to the generation of design implications. Therefore, this approach of considering implications before planning the fieldwork allows for an explicit selection of the study methodology which could offer the most relevant fieldwork: "if you get to the end of your study and think about implications, you have probably left it too late [...] I think about it right throughout the whole process as I set up experiments or observations" (P6).

\section{Evaluation Criteria of Implications for Design}

A particularly interesting finding is that design knowledge must address quality criteria pertaining to both scientific and design practice. These criteria consist of validity, generalizability and originality, as well as generativity, inspirability and actionability. Such criteria are particularly important but seldom met satisfactory by the generated implications for design. Although some people employ implicit criteria (P4,P10,P12), the importance of evaluating design knowledge is strongly acknowledged and the value of explicit criteria particularly emphasized (P6,P8,P7,P10,P11).

\section{Empirical Validity}

An important characteristic of design knowledge is empirical validity, supported through explicit accounts of how such knowledge is grounded in fieldwork data, or acquired through reflection on the evaluation of developed technologies. Being grounded in fieldwork data legitimates and provides rationale for design knowledge (P10,P11): "You should be able to trace back from implications and see how that's grounded" (P10). This requires articulating the explicit connection to the data: "those implications are self apparent [...] you need to be appropriate in terms and not making overly bold claims" (P6).

Testing design knowledge after implementation is perceived by some participants as the most important evaluation criterion, offering the strongest form of empirical validity (P2,P10,P12): "We developed a system and evaluated it and within that work the design implications really got better [...] the ability to build on implications is definitely a benchmark for evaluation; that's where one might find their value: in the extent someone could build on" (P10), as it provides the ultimate test: "building the system and evaluating it; that's the way that you can tell whether or not you've got the principles right" (P2). This suggests that imple- 
menting and testing design knowledge allows for the revision of design implications. It also builds on the idea that design implications are hypotheses whose value can be best proved through empirical tests: "implications are like hypotheses and the next thing in that process is to build a system that tests those hypotheses" (P12). A few participants also mentioned comparing their design implications against previous ones within an area, to ensure their consistency: "you are looking at different sources of literatures to see what kind of implications other people came up with" (P8). This also supports further critique, revision and extension of the existing body of design implications.

\section{Theoretical Validity}

Theoretical validity is supported through explanatory theories from human and social sciences. However, these sources for grounding design knowledge are seldom made explicit in the writings documenting their generation process. This criterion explicitly positions design implications as scientific knowledge that can be argued for, critiqued and revised: "check back to literature to see if you have support for your implications or not" (P8); as well as built on: "how the implications support the theories and concepts which are already out there" (P11) so that design researchers could "take the most salient findings and compare them to the spaces within the literature to point out how the implications that you are devising are relevant within the larger research space” (P10).

\section{External Validity: Generalizability}

Participants felt strongly that an important aspect of design implications is their generality when applied to settings beyond the fieldwork (P5,P6,P10). The challenge here resides in the differences between the settings which can reduce the applicability of implications (P5,P6). The issue of generalization from a single case study in qualitative research has been well studied and weaker forms have been accepted such as naturalistic generalization to similar settings [32] and generalization through aggregation of findings from multiple studies [31].

We found that participants employ both these forms with the former being the more frequent choice. In this case, abstractions are the preferred type of design implications as they promote generalization of findings from the situated fieldwork to other similar settings: "findings are very specific to your research question and then the implications are perhaps extrapolated slightly further from that to a more generalizable design space" (P10), "across a range of different [settings]" (P5). This suggests that an important property of abstractions is that they need to speak to multiple contexts, looking for example to "cut across different people, different households" (P1). In our data, there was however limited evidence regarding the exploration of the similarities of these settings.

Generalizations through aggregation of findings from independent studies was suggested through meta-abstractions on the basis of one's substantial body of work: "it takes a lot of activity in a domain [...] those design implications didn't come from a single user study, they came from multiple user studies, building multiple very different prototypes and kind of seeing what worked and what didn't work. So exactly where those abstract principles came from?" (P2). Such generalizations are the basis of meta-abstractions, and the aggregation is implicit. If such input from the previous body of work is made explicit, then the external validity of the generated implications could be strengthened.

\section{Originality}

Participants also emphasized the importance of originality, or developing implications that inform the design of novel technologies: "they describe a phenomenon that people didn't know about and could say: "You could build systems in this area, I hadn't thought about before" (P2). Ensuring originality is challenging, as generating design implications is difficult and supporting their novelty even harder (P7). Participants identified differences in the ability to generate original design implications relating to the maturity of the research area (P10), with newly emerging areas being better suited (P2). It also helps if there is general interest so that design implications can be built on by others: "you want multiple people to act on implications for design and sociologically, you need to have a bunch of people who are interested in something" (P2). People identified three sources of originality such as developing design implications that address a gap within the existing body of work: "identifying the salient findings and marking them against the gaps in the research" (P10); those which offer new perspectives on technology design: "tell a story that hasn't really been told so far" (P1); or those which explore new research areas: "At the time when we started doing that work it really wasn't a topic within HCI and we were able to start talking about those issues where other people haven't before" (P1). However, originality must also be tempered by usefulness, particularly when implications aim to inform the design of novel technologies: "Novelty is a bogus criterion because we could think of any number of bad novel systems" (P2).

\section{Generativity}

The exploration of new research areas for developing original design implications, points to another important criteria to evaluate design implications, namely their generativity. We defined this as the ability of design implications to create and open up new design spaces: "when implications for design appear really open and visionary, then I think that designers feel more freedom to become creative" (P11), and make them think differently: "when it comes to implications I'm looking for things that can inspire people to think in a different way" (P7). The generativity of design implications also relates to their ability to inform and inspire multiple instances of designs: "there are five or ten systems I could build from the abstract principles" (P2).

\section{Inspirability}

Design implications should also be inspirational in terms of motivating designers to explore them further or commit to implementing them: "That's interesting, I can think about 
some good concepts based on this" (P11). Being stimulating, interesting, provocative and engaging are all qualities that could contribute to the inspirational value. The right level of abstraction and ambiguity is also expected to open the design space: "something at a high enough level that can be inspiring [...] giving people room to apply it to their own systems" (P12). This implies that abstractions and sensitizing concepts are more inspirational than prescriptions, as they allow space for designers' creativity.

\section{Actionability}

Technological actionability implies that design implications are described in terms of technology properties, so that they can be implemented, or: "acted upon by technologists" (P2). Some participants expressed strong views on the importance of this quality; "I think that is fundamental that implications can be implemented: at least for part of them, there is something that you can act upon [...] implications for design means something that is practical, something that you can really operate on and I don't mean writing ideas for designs or design concepts but things that you can use in practical terms and it will give directions in that sense" (P4). For this, they need to be technologically feasible today or in the nearby future: "they should be implementable and they shouldn't be too futuristic" (P12). If design knowledge is not technologically actionable, it should be at least socially actionable by addressing the larger social setting where technologies are, could, or should (not) be employed. Such implications can be linked to technology by addressing aspects which may support or hinder technology adoption (P3), or specific technology goals: "You can imagine some sort of design intervention, which would be about awareness raising or about starting a political campaign to increase resources" (P9).

IMPLICATIONS FOR INTERACTION DESIGN RESEARCH

We now discuss the main findings and contributions of this work, highlighting several issues that design researchers may orient to while generating design implications. We suggest that researchers might benefit from understanding the different types of design implications, what constitutes support for generating them, how that knowledge needs to be actionable, general and inspirational. We also believe that the field can benefit from having a common language to characterize different types of design implications. Not least, the same criteria might be used to evaluate publications.

\section{Awareness of Different Types of Abstract Design Concepts}

Our findings reveal the prevalence of fieldwork-informed design concepts and a more nuanced understanding of this. Such design concepts are captured through four distinct types of design implications, i.e. abstract functionalities of new systems within existing technology classes, instantiations of such functionalities through design exemplars, sensitizing concepts as socially-oriented design concepts, and the new type of knowledge captured by meta-abstractions through abstract functionalities of new classes of technologies. More than half of identified design implications are represented by such design concepts. This is particularly important as design concepts are the only type of design knowledge that can be found on both sides of the gap, i.e. fieldwork and design practice. Subsequently, the more effort goes into generating these types of design implications, the greater the chances of better bridging the gap from fieldwork to design practice.

\section{Awareness of Technology Context and Social Categories} In terms of generating design implications, our findings extend previously identified sources such as fieldwork data, design practice and human science theories. A striking finding is the emphasis on the value of technology context, described as the awareness of affordances and limitations of existing and in particular cutting-edge technologies. Another novel source for generating design implications is existing HCI work and in particular the corpus of design implications developed in a specific HCI area. We found limited reference to human science theories as a source of generating implications, but strong evidence for the use of specific social categories such as social values, norms, time and place. While our findings show limited references to the practice-informed design implications, this has been emphasized as a particularly relevant source for generating or revising meta-abstractions.

Awareness of Heuristics for Generating Design Knowledge The process of generating implications extends the traditional approach of developing implications after concluding fieldwork analysis [1,10,11]. In contrast, novel ways of generating design implications occur during the fieldwork analysis, or even by considering implications while planning the fieldwork study. This latter approach captures the importance of technology context not only as a source of design implications but also in preparing and selecting social settings to be explored, if they are to more successfully inform the generation of design implications.

Provide Evidence to Support Validity of Design Implications Another important outcome is that design implications aim to meet the validity criteria of scientific knowledge. However, current practice provides limited evidence for validity. Our findings reveal a large range of sources of design implications which can not only support the generation process but also its scrutiny and critique. Sensitivity to these sources of information and researchers' efforts for making them explicit are much needed to strengthen the validity of design implications. For example, empirical validity could be substantiated by grounding design knowledge in the fieldwork data or even better by building and evaluating previously designed technologies. Theoretical validity is supported by grounding the design knowledge in social sciences theories. External validity could be claimed by exploring the similarities between multiple settings or making explicit the findings from multiple studies employed as design resources.

\section{Specify Generalizable Actionable Knowledge}

Our findings also point to the importance of actionable knowledge embedded in the implications for design. The 
more situated and concrete this knowledge is, the closer to requirements it gets. But requirements lack both abstraction and ability to generalize. A specific useful combination of two types of design implications supporting design practice, is abstract functionalities combined with their instantiations. This combination is actionable because it provides concrete design concepts through instantiations. Moreover, it is also generalizable because the abstraction can be applied across multiple situations. Together, this combination provides a powerful device for bridging situated and general settings.

\section{Provide Inspirational and Generative Power}

A specific new value of design implications is that they should call for action, motivating designers to engage in their exploration. We need to generate implications that are engaging, compelling designers to explore them further by fostering broad divergent thinking. Future work should explore the characteristics of compelling implications and how they could support creative thinking or designers' emotions and values [22].

\section{CONCLUSIONS}

The main contribution of this work is a participant derived taxonomy to describe design implications. The taxonomy offers a framework for reflecting, debating and conversing within this challenging area, as well as documenting values that different types of design implications might serve. Various heuristics supporting the process of generating implications and a set of criteria for evaluating such knowledge offer much needed insight into a current and relevant research topic.

\section{ACKNOWLEDGMENTS}

This work was supported by the Swedish Govern. Agency for Innovation Systems to the Mobile Life VinnExcellence Centre, in partnership with Ericsson, Microsoft, Nokia, IKEA and the City of Stockholm (2011-03460).

\section{REFERENCES}

1. Ackerman, M.S. The intellectual challenge of CSCW: the gap between social requirements and technical feasibility. Human.Computer Interaction. 15, 2 (2000), 179-203.

2. Alexander, C., Ishikawa, S., \& Silverstein, M. (1978). A Pattern Language: Towns, Buildings, Construction.

3. Benyon, D. R., Turner, P. \& Turner, S. Designing Interactive Systems. Pearson Education, London, UK, 2005.

4. Beyer, H., and Holtzblatt, K. Contextual Design. Morgan Kaufmann, San Francisco, CA, USA, 1998.

5. Blumer, H. What is wrong with social theory? American Sociological Review 19, 1 (1954), 3-10.

6. Carroll JM, ed. 1991. Designing Interaction: Psychology at the Human-Computer Interface. NY: Cambridge Univ. Press

7. Carroll, J. M. \& Kellogg, W. A. Artifact as theory-nexus: hermeneutics meets theory-based design. Proc.CHI 1988. ACM (1989), 7-14.

8. Carroll, J.M. (ed) Scenario-Based Design: Envisioning Work and Technology in System Development. 1995.

9. Cooper, A. The Inmates are Running the Asylum. SAMS, 1999.

10. Crabtree, A. and Rodden, T. Ethnography and design? Proc. IAISCR (2002) 70-74.
11. Crabtree, A., O’Brien, J., Nichols, D., Rouncefield, M., and Twidale, M. Ethnomethodologically informed ethnography and information systems design. JASIST, 51, 7, (2000), 666-682.

12. Dix, A., Finlay, J., Abowd, G.D. and Beale, R. Human-computer interaction. Pearson Prentice, New York, NY, USA, 2004.

13. Dourish, P. Implications for design. Proc.CHI 2006, ACM (2006), 541-550.

14. Dourish. P. and Bellotti.V. Awareness and coordination in shared workspaces. Proc. CSCW 1992. ACM (1992), 107-114

15. Fallman, D., Design-oriented human-computer interaction. In Proc.CHI 2003, ACM (2003), 225-232.

16. Gaver, B., \& Martin, H. Alternatives: exploring information appliances through conceptual design proposals. In Proc. CHI 2000,_ACM (2000),_209-216.

17. Gaver, W.W., Beaver, J., \& Benford, S. Ambiguity as a resource for design. In Proc. CHI 2003. ACM (2003), 233-240.

18. Gray, W. D., \& Salzman, M. C. (1998). Damaged merchandise? Human-Computer Interaction, 13(3), 203-261.

19. Grudin, J. and Pruitt, J. Personas, participatory design and product development: an infrastructure for engagement. In Proc. PDC 2002, (2002), 144-161.

20. Haynes, S. R. and Carroll, J. M. (2008). Theoretical Design Science in HCI: A Practical Concern? Artifact, 1(3), 159-171.

21. Höök, K. and Löwgren. J. 2012. Strong concepts: Intermediate-level knowledge in interaction design research. ACM ToCHI. 19, 3, 23.

22. Hughes, J.A., O'Brien, J., Rodden, T., Rouncefield, M., and Blythin, S. Designing with ethnography: a presentation framework for design. Proc. DIS 1997, ACM (1997), 147-158

23. Hughes, J.A., Randall, D., and Shapiro, D. Faltering from ethnography to design. Proc. CSCW 1992. ACM (1992), 115-122.

24. Johnson, J., Roberts, T. L., Verplank, W., Smith, D. C., Irby, C. H., Beard, M., \& Mackey, K. (1989). The Xerox star: A retrospective. Computer, 22(9), 11-26.

25. Luff, P., Heath, C., Kuzuoka, H., Yamazaki, K., and Yamashita, J. Handling documents and discriminating objects in hybrid spaces. Proc. CHI 2006. ACM (2006), 561-570.

26. Macaulay, C., Benyon, D. and Crerar, A. Ethnography, theory and systems design: from intuition to insight, IJHCS 53, 1, (2000), 35-60.

27. Norman, D. A. and Draper, S. W. User Centered System Design: New Perspectives on HCI. Erlbaum, Hillsdale, 1986.

28. Paay, J. (2008). From ethnography to interface design. Handbook of Research on User Interface Design and Evaluation for Mobile Technology, 1-15.

29. Rittel H, Webber M. 1973. Dilemmas in a general theory of planning. Policy Sci. 4: 155-69

30. Sas, C and Zhang, C. Do emotions matter in creative design? Proc. DIS 2010. ACM (2010), 372-375.

31. Schofield, J. W. (2002). Increasing the generalizability of qualitative research. The qualitative researcher's companion, 171-203.

32. Stake, R. E., Trumbull, D. J. (1982). 1 Naturalistic Generalizations.

33. Taylor, A. (2009). Ethnography in Ubiquitous Computing. In Ubiquitous Computing Fundamentals, 203-236. Chapman \& Hall.

34. Vallgårda, A., \& Redström, J. Computational composites. In Proc.CHI 2007. ACM 2007, 513-522.

35. Yamashita, N., Kaji, K., Kuzuoka, H., and Hirata, K. Improving visibility of remote gestures in distributed tabletop collaboration. Proc. CSCW 2011. ACM Press (2011) 95-104.

36. Zimmerman, J., Stolterman, E., and Forlizzi, J. An analysis and critique of Research through Design: towards a formalization of a research approach. Proc. DIS 2010. ACM (2010), 310-319. 